\title{
Understanding Flow of Energy in Buildings Using Modal Analysis Methodology
}

\section{ES-FuelCell 2013}

\author{
John Gardner \\ Kevin Heglund \\ Kevin Van Den Wymelenberg \\ Craig Rieger
}

\author{
July 2013
}

The INL is a

U.S. Department of Energy

National Laboratory

operated by

Battelle Energy Alliance

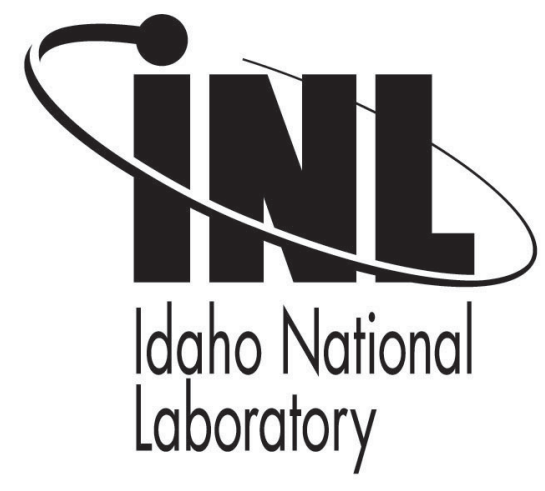

This is a preprint of a paper intended for publication in a journal or proceedings. Since changes may be made before publication, this preprint should not be cited or reproduced without permission of the author. This document was prepared as an account of work sponsored by an agency of the United States Government. Neither the United States Government nor any agency thereof, or any of their employees, makes any warranty, expressed or implied, or assumes any legal liability or responsibility for any third party's use, or the results of such use, of any information, apparatus, product or process disclosed in this report, or represents that its use by such third party would not infringe privately owned rights. The views expressed in this paper are not necessarily those of the United States Government or the sponsoring agency. 
Proceedings of the ASME $20137^{\text {th }}$ International Conference on Energy Sustainability \& $11^{\text {th }}$ Fuel Cell Science,
Engineering and Technology Conference
ES-FueICell 2013
July 14-19, 2013, Minneapolis, MN, USA

ES-FuelCell2013-18390

\section{UNDERSTANDING FLOW OF ENERGY IN BUILDINGS USING MODAL ANALYSIS METHODOLOGY}

\author{
John Gardner \\ Boise State University \\ Boise, ID, USA \\ Kevin Van Den Wymelenberg \\ University of Idaho \\ Boise, ID, USA
}

\author{
Kevin Heglund \\ Boise State University ${ }^{1}$ \\ Boise, ID, USA
}

\begin{abstract}
It is widely understood that energy storage is the key to integrating variable generators into the grid. It has been proposed that the thermal mass of buildings could be used as a distributed energy storage solution and several researchers are making headway in this problem. However, the inability to easily determine the magnitude of the building's effective thermal mass, and how the heating ventilation and air conditioning (HVAC) system exchanges thermal energy with it, is a significant challenge to designing systems which utilize this storage mechanism. In this paper we adapt modal analysis methods used in mechanical structures to identify the primary modes of energy transfer among thermal masses in a building. The paper describes the technique using data from an idealized building model. The approach is successfully applied to actual temperature data from a commercial building in downtown Boise, Idaho.
\end{abstract}

\section{INTRODUCTION}

Modeling building energy use has a long history in architecture and engineering. These modeling efforts can be viewed along a spectrum which is anchored on one end by firstprinciple, ground-up models (such as those implemented in EnergyPlus,(EERE 2012)). On the other end of the spectrum are so-called "black box" models which use actual building energy consumption data to derive correlations with exogenous

\footnotetext{
${ }^{1} \mathrm{~K}$. Heglund is currently with PAR Electric in Billings, MT
}

factors like daily temperature, sunshine and occupancy (Ahmad and Culp 2006; Wijayasekara, Manic et al. 2012). Modeling activity on both ends of the spectrum has increased dramatically in recent years. Building designers, motivated by client demands and third party certifications (such as Leadership in Energy and Environmental Design (LEED)) are increasingly using first principle building energy models as part of the building design process. These models are valuable tools in assessing various energy savings options, comparing initial cost with likely energy savings and computing the cost-effectiveness of each choice. These models, however, have little utility in understanding the operation of the building after it is built.

On the other end of the spectrum, the proliferation of smart meters and network-enabled sub-meters has made available an unprecedented amount of data which can be used to develop increasingly complex and detailed black box models.

Between the two extremes one can find a series of approaches that begin with some physics-based model of energy storage (thermal mass) and resistance to heat flow (insulation) and perhaps mass transfer, coupled with a parameter identification technique that utilizes energy consumption data to fit parameter values to the physics models. This family of approaches is often described as "gray box" models.

A closely related field is the application of system identification methods to find values of model parameters for building energy use models (Ljung 1997). These approaches are important for models which have some basis of physics in the modeling approach(Mejri, Barrio et al. 2011). 
A detailed survey of black box modeling methods, and their relative effectiveness in predicting energy use from a real data set can be found in (Kreider and Haberl 1994). This paper presents the results of an open competition to find the best methods in utilizing building energy data to develop a predictive building energy use model. The methodologies that were used include nonlinear statistical models, various Artificial Neural Network topologies and piecewise linear regressions. Prediction methods were judged by two statistical measures, a coefficient of variation (a root mean square error) and mean bias error. These measures were applied to each sub-set of building energy measure (electric, chilled water, hot water) and to overall energy consumption. While the various methods each had their strengths, none was considered uniformly superior for all indices of performance.

The term "modal analysis" refers to a set of linear algebrabased tools that can be used to model, visualize and understand the vibrational behavior of complex mechanical structures. Using eigenvalues and eigenvectors of a system of differential equations, engineers can identify the frequencies at which a structure vibrates and the relative displacements, or shapes, of the structure as it vibrates at each frequency. These tools are particularly powerful in the context of laboratory or in-field testing of existing structures. In particular, modal analysis offers a means of finding a relatively low-order dynamic model of highly complex systems.

\section{METHODOLOGY}

The central theme in gray box modeling is finding the best model structure for a given application. Typically a good model structure is one that is complex enough to capture significant dynamic behavior, but no more complex than is required to explain the relevant system behavior. The simplest such model for building energy consumption is described in (Rabl 1988) as a 1R1C network, in essence, a single lumped thermal mass surrounded by a thermal resistance (i.e. insulation). Equation 1 is the differential equation for such a model.

$$
C \dot{T}=K\left(T_{\infty}-T\right)
$$

Where $\mathrm{C}$ is the thermal capacitance (i.e. thermal mass) at temperature $\mathrm{T}, \mathrm{K}$ is the thermal conductance (reciprocal of resistance) of the insulating envelope and $T_{\infty}$ is the environmental temperature.

Clearly, a model of this level of simplicity, ignoring temperature variations throughout the structure, air exchanges, HVAC energy additions \& removals and internal loads, just to name a few, is of limited utility. However, it is possible to extract useful information from even this limited approach.

Extending this model to one in which there are several thermal masses exchanging heat with the environment and each other, through different thermal transmittances, we have a more general and $\mathrm{N}$-dimensional model.

$$
C_{n} \dot{T}_{n}=\sum_{k=1}^{N} K_{n k} T_{k}+\sum_{i=1}^{M} H_{n i} U_{i}
$$

Which can be represented in matrix form as shown

$$
\mathbf{C} \underline{T}=\mathbf{K} \underline{T}+\mathbf{H} \underline{U}
$$

Where $\mathrm{C}$ is a diagonal, positive definite matrix of the thermal masses, $\mathrm{K}$ is a symmetric matrix of thermal conductances, $\mathrm{U}$ is the vector of inputs, and $\mathrm{H}$ is an input matrix.

Since $\mathrm{C}$ is positive definite (and is hence invertible), the state-space form of equation (2) is easily shown to be

$$
\dot{T}=\mathbf{C}^{-1} \mathbf{K} \underline{T}+\mathbf{C}^{-1} \mathbf{H} \underline{U}
$$

In the special case where the observed temperatures are also the system states, then the output equation is trivial. But that is rarely the case, and in actual applications, the relationship between the observed temperatures and the system states is unknown. Indeed, this relationship lies at the heart of the system identification process.

In more general form, the dynamics are determined by the states of the system given by the vector $\mathrm{Q}$ in equation (5).

$$
\begin{aligned}
& \dot{Q}=\mathbf{A} \underline{Q}+\mathbf{B} \underline{U} \\
& \underline{T}=\mathbf{C} \underline{Q}+\mathbf{D} \underline{U}
\end{aligned}
$$

Where $\mathrm{Q}$ is the $\mathrm{N}$-dimensional state vector, $\mathrm{T}$ is the $\mathrm{L}$ dimensional output vector which corresponds to the measured, or observed temperatures and $\mathrm{U}$ is the $\mathrm{M}$-dimensional input vector. D is the "direct transmission" matrix, typically zero but included here for the sake of completeness.

The eigenvalues and eigenvectors of the system matrix, $\mathbf{A}$, allow for a transformation of (5) into a diagonalized (or decoupled) set of equations.

$$
\begin{aligned}
& \underline{\dot{X}}=\mathbf{E} \underline{X}+\mathbf{B}^{\prime} \underline{U} \\
& \underline{T}=\mathbf{C P} \underline{X}+\mathbf{D} \underline{U}
\end{aligned}
$$

Where $\mathrm{E}$ is the diagonal vector of eigenvalues and:

$$
\begin{aligned}
& \mathbf{E}=\operatorname{eig}(\mathbf{A}) \\
& \underline{Q}=\mathbf{P} \underline{X} \\
& \mathbf{B}^{\prime}=\mathbf{P}^{-1} \mathbf{B}
\end{aligned}
$$


And $\mathbf{P}$ is the orthonormal matrix made up of the eigenvectors of the system matrix $\mathbf{A}$ (arranged in columns). The eigenvalues in $\mathbf{E}$ are reciprocals of time constants that describe the response of the system (Rabl 1988; Lefebvre 1997; Matiasovsky 2006).

The form of the dynamic equations shown in (6) is known as the modal form. As is the case with structural modal analysis, model reduction can often be realized by considering only the slowest modes, as the majority of any dynamic response can be described by a subset of the entire system.

Therefore, the method required to carry out an analysis of a system can be described in the steps below:

1) Given a set of $L$ temperatures sampled over a period of time, and a set of $\mathrm{M}$ input variables sampled over the same period

2) Use system identification methods to identify a state space model of the form shown in equation (5) that captures the dynamic relationship between the inputs and observations to an adequate level. This step can be iterated to find the minimum order of model that adequately describes the system behavior.

3) Put the system into modal form as seen in equation (6)

4) Interpret system behavior by examining the eigenvectors and eigenvalues.

If the system under study is a passive system such as the one described in equation (1), all the eigenvalues of the system will be real and no oscillatory behavior is possible. However, if the process is applied to a building with active HVAC control (where oscillatory behavior is common), then the process will likely identify one or more modes that are complex and the eigenvalues will appear as complex conjugate pairs, which represent the frequency of oscillation.

\section{APPLICATION OF METHOD: SYNTHETIC DATA}

To demonstrate the process, a simple model is constructed and used to generate synthetic temperature readings through simulation. Those readings are then treated like measured data to derive the various eigenvalues and eigenvectors (time constants and mode shapes) as described above. Since we are starting with an analytical model with known parameter values, we can compare the results of the modal modeling process with the correct answers to ensure that the process is internally consistent.

Figure 1 shows an idealized building model consisting of 9 adjacent, but insulated spaces, each space is a thermal mass, each adjacency has a thermal resistance. The outside of the square is at ambient temperature.

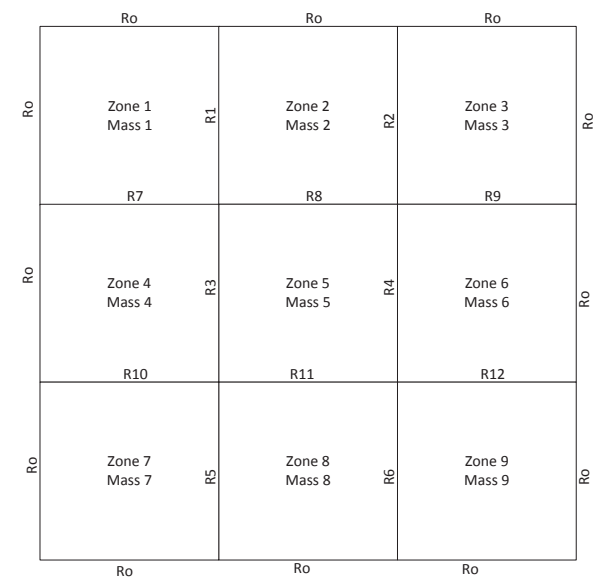

Figure 1: Schematic representation of the nine thermal mass model used to demonstrate the method

To utilize the 9 zone model described above generic values for $R_{0}$ to $R_{12}$ and $M_{1}$ to $M_{9}$ were arbitrarily chosen and the inputs to the model were taken to be the outside temperature data (taken from actual weather data) and estimated occupancy schedule. The actual eigenvalues and mode shapes are derived using analytical and numerical means. These values could then be compared to the estimated time constants and mode shapes that were derived using the functions built into MATLAB ${ }^{\circledR}$ system identification toolbox to estimate State Space models.

Table 1: Parameter values used to generate synthetic data in 9-

\begin{tabular}{|l|r|}
\hline $\mathrm{R}_{1}$ & 12 \\
\hline $\mathrm{R}_{2}$ & 9 \\
\hline $\mathrm{R}_{3}$ & 20 \\
\hline $\mathrm{R}_{4}$ & 6 \\
\hline $\mathrm{R}_{5}$ & 5 \\
\hline $\mathrm{R}_{6}$ & 17 \\
\hline $\mathrm{R}_{7}$ & 9 \\
\hline $\mathrm{R}_{8}$ & 4 \\
\hline $\mathrm{R}_{9}$ & 5 \\
\hline $\mathrm{R}_{10}$ & 91 \\
\hline $\mathrm{R}_{11}$ & 12 \\
\hline $\mathrm{R}_{12}$ & 9 \\
\hline $\mathrm{R}_{0}$ & 30 \\
\hline
\end{tabular}
zone model

Two inputs drive this system. One is the outside temperature which is taken from actual weather observations. The other is a binary function which corresponds to the "occupied" state of the building. For the purposes of this example, it serves as the proxy of the building HVAC system and is seen by the system as a heat source for the thermal masses. Figure 2 shows the time history of the two input functions which will be used for this demonstration. 

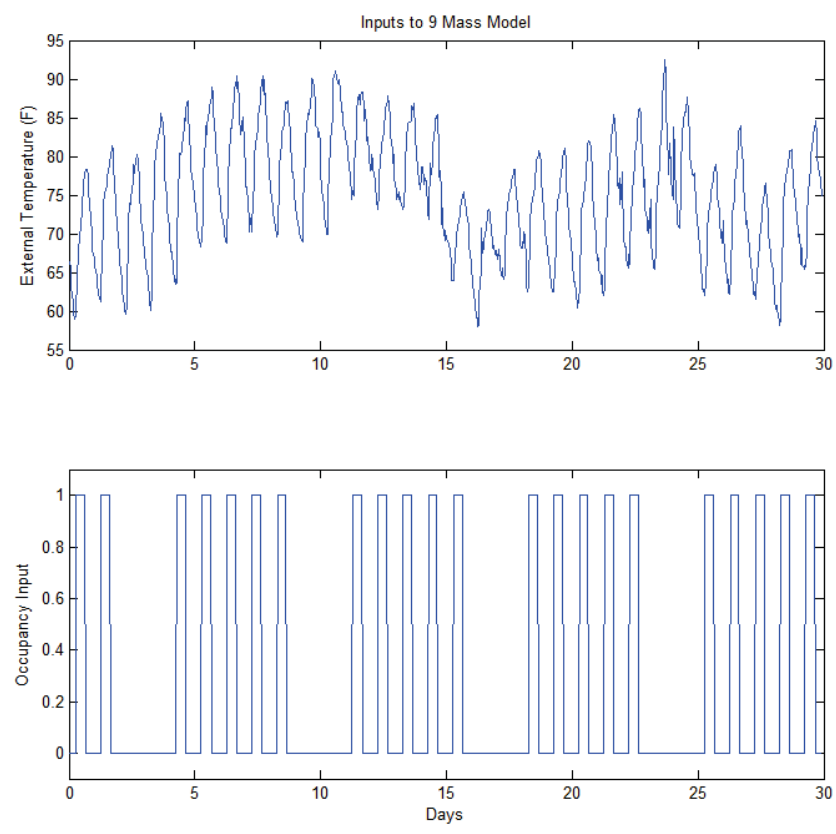

Figure 2: Inputs to 9 zone model are external temperature and an occupancy marker (value of 1 between 8 am and 5pm).

Applying a simple energy balance to each thermal mass results in 9 first order differential equations that can be put in the form of equation (5) and diagonalized to the modal form (6). Table 2 shows the time constants (reciprocals of the eigenvalues) and the eigenvectors for each time constant.

Table 2: Time constants and eigenvectors for the 9-zone model

Modes

Time Constant (Min)

\begin{tabular}{|r|r|r|r|r|r|r|r|r|}
\hline 1 & 2 & 3 & 4 & 5 & 6 & 7 & 8 & 9 \\
\hline 0.15 & 0.49 & 0.63 & 1.14 & 1.21 & 1.88 & 21.35 & 10.43 & 4.63 \\
\hline
\end{tabular}

Zone 1

Zone 2

Zone 3

Zone 4

Zone 5

Zone 6

Zone 7

Zone 8

Zone 9

\begin{tabular}{|r|r|c|c|c|c|c|c|c|}
\hline-0.02 & 0.21 & 0.11 & -0.57 & -0.85 & 0.26 & 0.33 & 0.35 & 0.05 \\
\hline 0.33 & -0.78 & -0.24 & -0.03 & -0.20 & 0.36 & 0.29 & 0.08 & 0.29 \\
\hline-0.03 & 0.27 & -0.72 & 0.04 & 0.22 & 0.49 & 0.27 & 0.01 & 0.47 \\
\hline 0.01 & 0.00 & -0.01 & 0.05 & 0.09 & -0.07 & 0.43 & 0.58 & -0.14 \\
\hline-0.94 & -0.51 & 0.11 & 0.14 & -0.08 & 0.25 & 0.31 & 0.03 & 0.29 \\
\hline 0.06 & 0.08 & 0.61 & 0.03 & 0.22 & 0.25 & 0.28 & -0.02 & 0.49 \\
\hline 0.00 & -0.01 & 0.00 & -0.17 & 0.08 & 0.04 & 0.42 & -0.60 & -0.21 \\
\hline 0.03 & 0.07 & 0.00 & 0.75 & -0.35 & -0.08 & 0.36 & -0.38 & 0.05 \\
\hline 0.00 & -0.02 & -0.13 & -0.25 & -0.02 & -0.65 & 0.29 & -0.16 & 0.54 \\
\hline
\end{tabular}

It is instructive to take a moment to consider the implications of the values and vectors in Table 2. The first column describes a mode of the system where the time constant (in minutes) describes how fast the temperature changes. For example, a system with at time constant of 1 minute subjected to step change if its input will be settle to within $5 \%$ of its new temperature within 3 minutes (and less than $1 \%$ in 5 minutes). The column shows us how the various zones participate in this mode. For the mode in column 1, we can see that zone 5 dominates this mode with the largest absolute value. Second most import zone is 2 . All the others zones have very small contributions. Note also that the signs of the two largest contributions are opposite. We interpret this by noting that there is a mechanism of heat transfer that affects zones 2 and 5 in such a way that the fundamental dynamics have a time constant of 0.15 minutes and that the temperature fluctuations described by this mode are in the opposite directions (i.e. one is warming while the other is cooling).

Note that each zone participates in each mode, so the actual temperature record is a linear combination of the various mode responses.

In structural systems, mode shapes (eigenvectors) are often visualized by imposing color on an image of the structure where the color value indicates the value of that particular element in the eigenvector. For this example, we arbitrarily choose red for positive values and blue for negative (there is no significance to the sign, only relative to the other elements). The intensity of the color is an indication of where the absolute value of the element lies. Paler colors close to 0 , brightest close to 1 .

Figure 3 shows the graphical representative of the $7^{\text {th }}$ mode of this model.

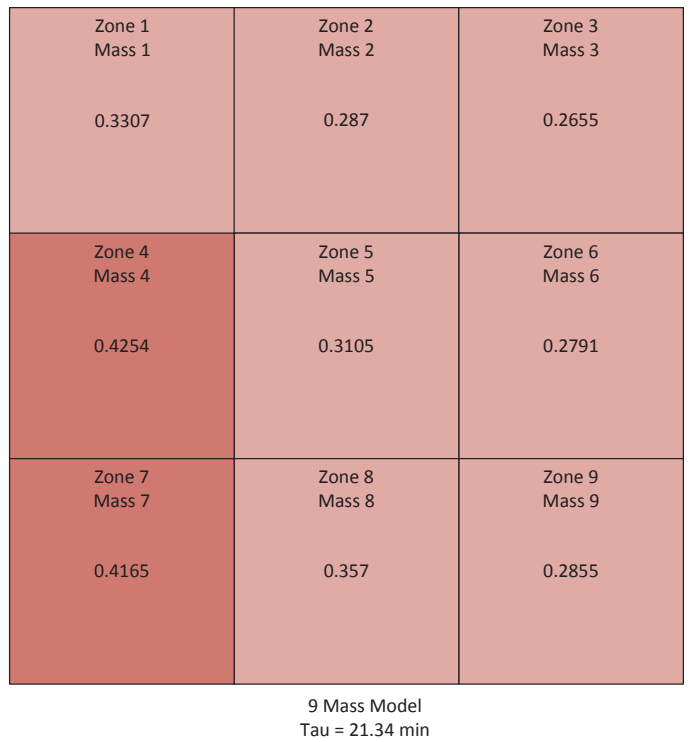

Figure 3: Seventh mode shape for the 9 mass model (time constant $=21.34 \mathrm{~min}$ )

Note that this mode is the slowest of the modes and shows a relatively uniform response of the masses. The likely interpretation is that this shows the distribution of the thermal energy after all other transients have settled out.

Figure 4 shows the graphical representation of the next slowest mode, showing a much more complex behavior. 


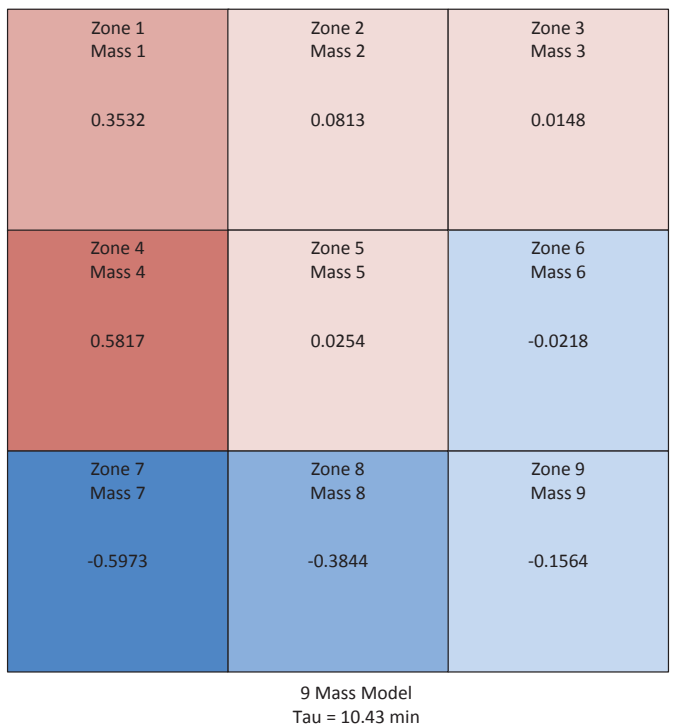

Figure 4: The $8^{\text {th }}$ mode of the 9 mass model at a time constant of $10.43 \mathrm{~min}$.

To verify the utility of this method in identifying the parameters from measured data, the differential equations for the nine mass model were numerically solved (using Simulink) with the signals shown in Figure 2 as the driving inputs. The simulation generated temperature records of the nine zones which can then be used as input to the system identification process.

Figure 5 shows the time record that results from the simulation.

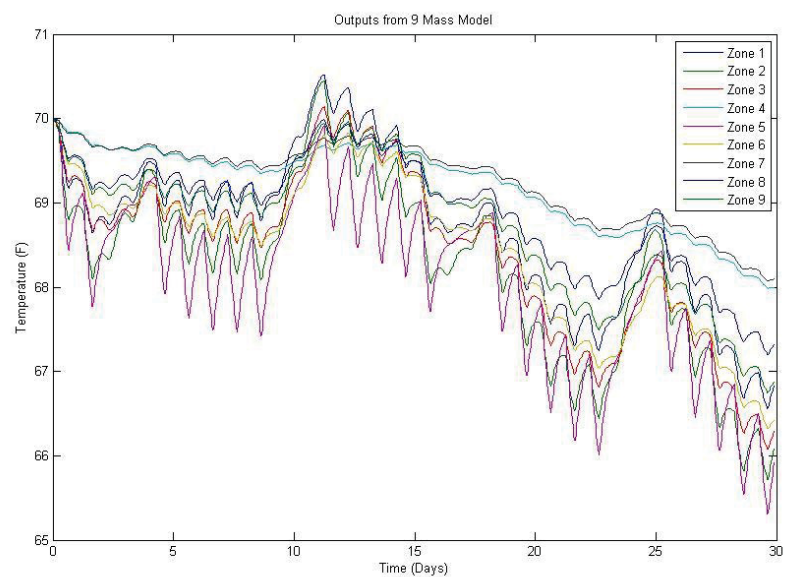

Figure 5: The temperature of each zone of the 9 mass model, corresponding to the inputs from Figure 2.

Using the MATLAB System Identification Toolbox, a variety of models of any order can be fit to the data. In particular, we focus on state space models of the form seen in equation (5). The advantage of this approach is that it becomes very easy to explore the accuracy of reduced order models.
Figure 7 is a screen shot of the SI toolbox comparing the fit for models of order 1, 2, 3, 8 and 9. Note that while the first order model is (understandably) a poor fit, all the other models provide reasonable approximations.

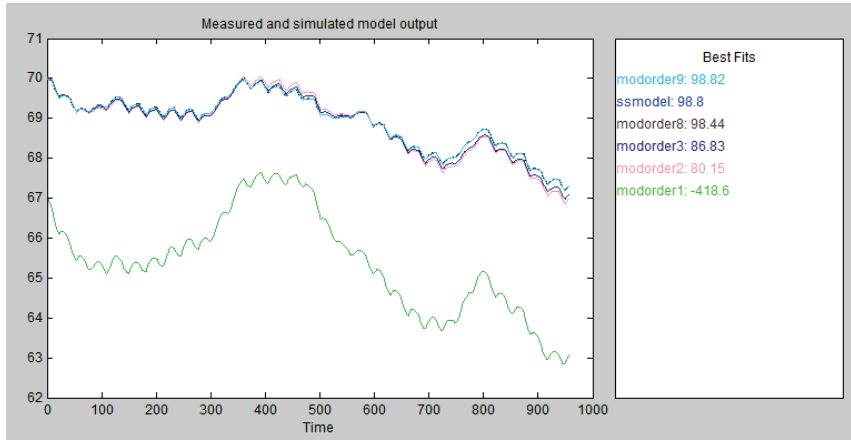

Figure 6: System Identification GUI showing the fit between model orders 1, 2, 3, 8, 9, the original model to the actual data derived from Simulink on Zone 8. Note that model order 1 is a poor fit, but model order 2 is greatly improved. It can also be seen that model orders 8, 9, and the original model fit the data nearly perfectly.

To explore how effective this approach was in identifying the modes of the original model, we generated the color plots for a few modes and compare them to the plots generated from the original model. Figure 7 shows the two plots for the $9^{\text {th }}$ mode (time constant 4.63 minutes). As one might expect, the system identification approach, using a full-order model with perfect data, was able to back out the correct answer.
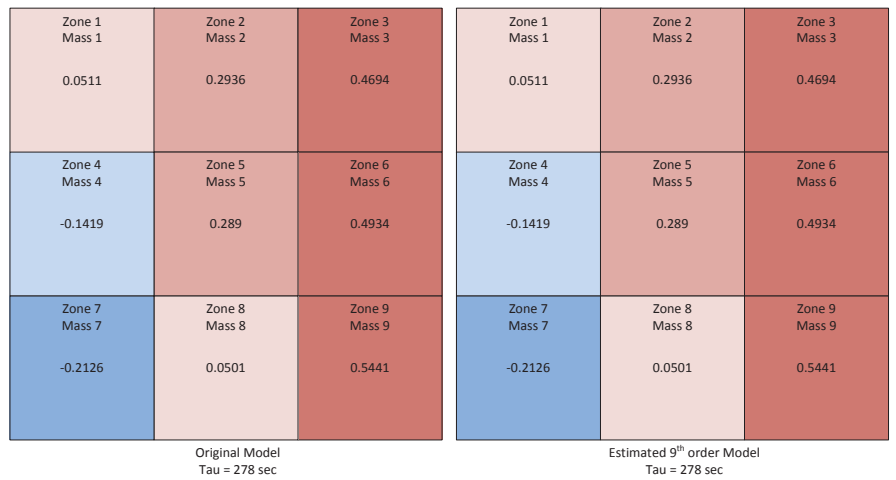

Figure 7: Modal shapes of the original model and the $9^{\text {th }}$ order estimated model. The $9^{\text {th }}$ order estimated model directly relates to the original model (down to the $4^{\text {th }}$ decimal point).

Figure 8 shows the results of a reduced order model $\left(7^{\text {th }}\right.$ order $)$ in identifying the second mode in Table 2 (time constant 0.49 minutes) to a high degree of accuracy. 

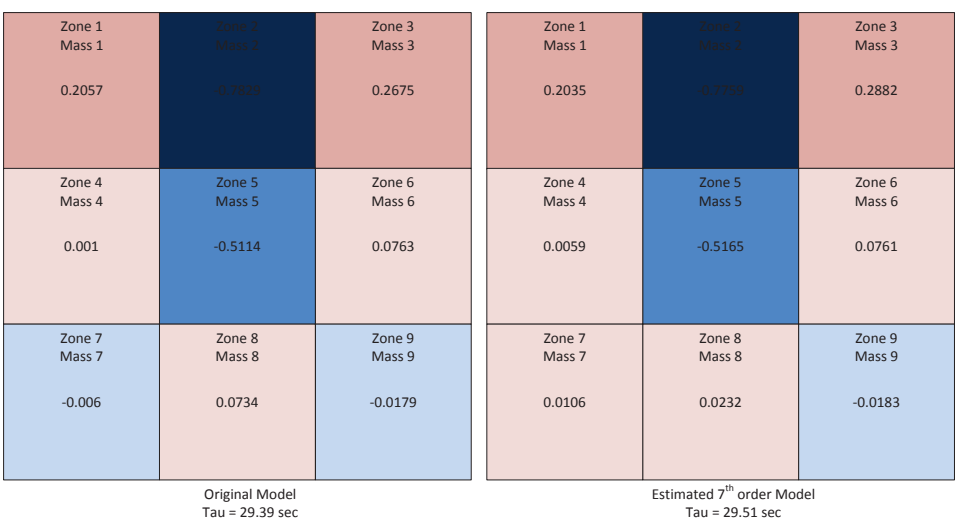

Figure 8: Modal shapes of the original model and the $7^{\text {th }}$ order estimated model

\section{APPLICATION TO ACTUAL BUILDING DATA}

The technique can be demonstrated with actual data as well. For this purpose, the time history of building zone temperatures were obtained by querying the building energy control system and transferring them to a database where they can be manipulated.

The candidate building is multi-floor office building in downtown Boise, Idaho. The data were supplied for two floors (floors 5 and 7) as seen in Figure 9. The length of monitoring time was a 1 month period (September 2011). The data supplied contained temperatures for each zone on the two floors, as well as outside temperature. For the purposes of system identification, the input data were taken to be the outside temperature and an occupancy signal that corresponded to 8 $\mathrm{AM}$ to $5 \mathrm{PM}$ on weekdays. This is the same input data used in the demonstration in the previous section (Figure 2).

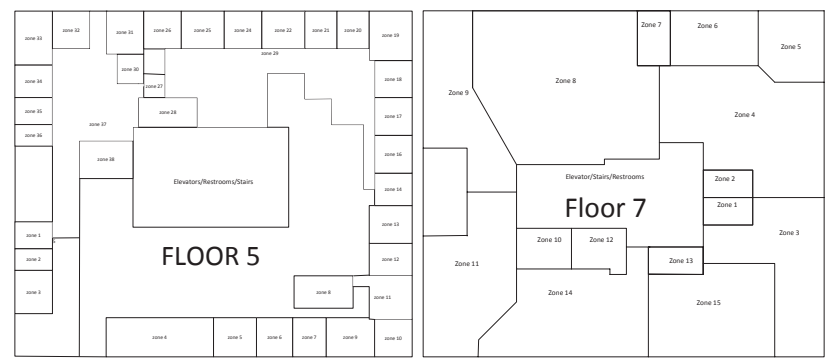

Figure 9: Floorplan of Floors 5 and 7. There are 38 zones on floor 5 and 15 zones on floor 7 . The building is oriented nearly $45^{\circ}$ from the compass points, with the upper-right corner facing approximately south

\section{$\underline{7^{\text {th }} \text { Floor }}$}

By utilizing the system identification toolbox and the techniques described for the 9 mass system model, state space estimations were identified. This was performed using different model orders to estimate the fit. In establishing the best model for the $7^{\text {th }}$ floor, decreasing orders of fit were attempted. It was noted that model fidelity was not dramatically impacted until the model order was reduced to $5^{\text {th }}$ order. Therefore, a $6^{\text {th }}$ order model was chosen to represent the data set with 15 individual temperature readings.

Figure 10 shows a screen shot of the toolbox for a week-long period of data and several models that were computed.

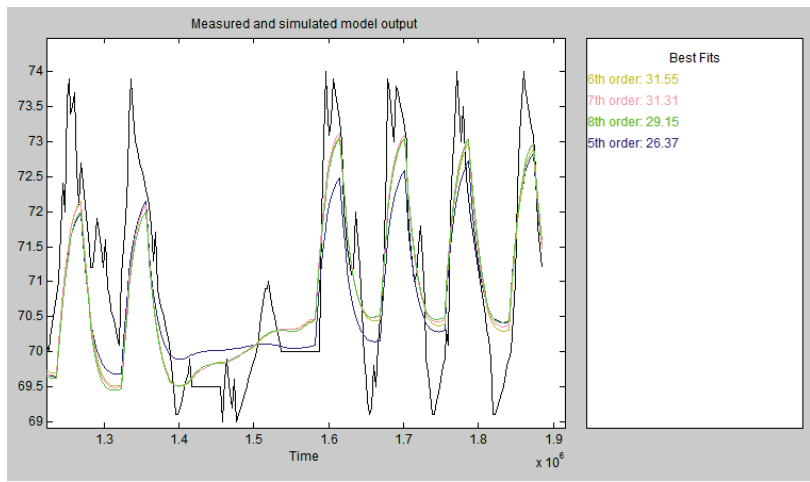

Figure 10: Different order models fit to Floor 7 -Zone 2 of the office building. This is a fairly good fit for this data.

Once a reasonably suitable state space model is identified (of the form given in equation (5)), the eigenvalues and eigenvectors of the system matrix (A) can be found. As discussed earlier, it is likely that an analysis like this, where no attempt is made to tease out the HVAC control system behavior from the passive thermal behavior of the building structure, complex eigenvalues are likely to be observed. This correlates to a mode shape that is oscillatory and not simply decaying with time. These values appear as complex conjugate pairs.

Finally, we note that the System Identification Toolbox allows the option of fitting a diagonal state space model. In essence, it chooses states that are already the modes of the system, and a system matrix that is already diagonalized.

Figure 11 shows the readout of the 6th order model that was derived from the 15 temperature readings from the $7^{\text {th }}$ floor of the building. 


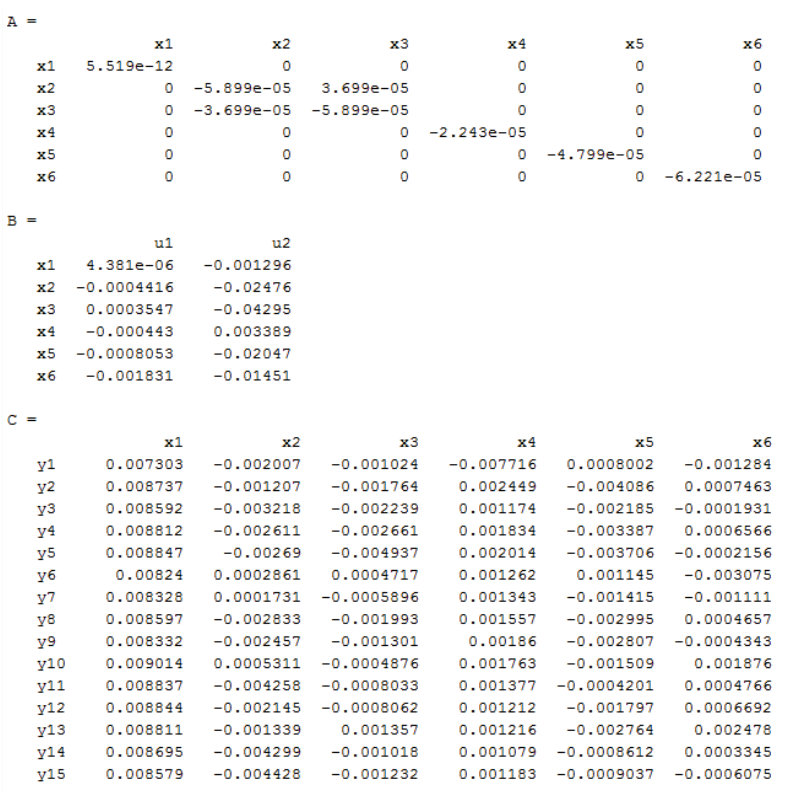

Figure 11: A $6^{\text {th }}$ order model of the $7^{\text {th }}$ floor of the office building.

Note that the system matrix is not entirely diagonal, the $2^{\text {nd }}$ and $3^{\text {rd }}$ columns and rows each have one off-diagonal entry. This is how complex eigenvalues arise in this technique. One additional step is required to resolve that particular mode to the special case where the mode represented is oscillatory. Figure 12 shows the eigenvalues properly transformed showing the complex conjugate pair. These can be interpreted as a complex conjugate pole where their magnitudes correspond to the natural frequency and their imaginary part correspond to the actual frequency of oscillation.

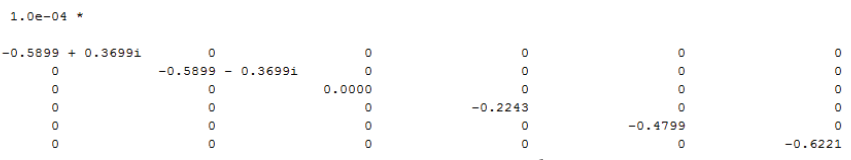

Figure 12: The eigenvalues of the $6^{\text {th }}$ order model above.

Due to the existence of the oscillatory mode there are 5 unique mode shapes for the $6^{\text {th }}$ order model. Figures 13 through 17 show graphical representation of the mode shapes using the same methodology demonstrated in the previous section.

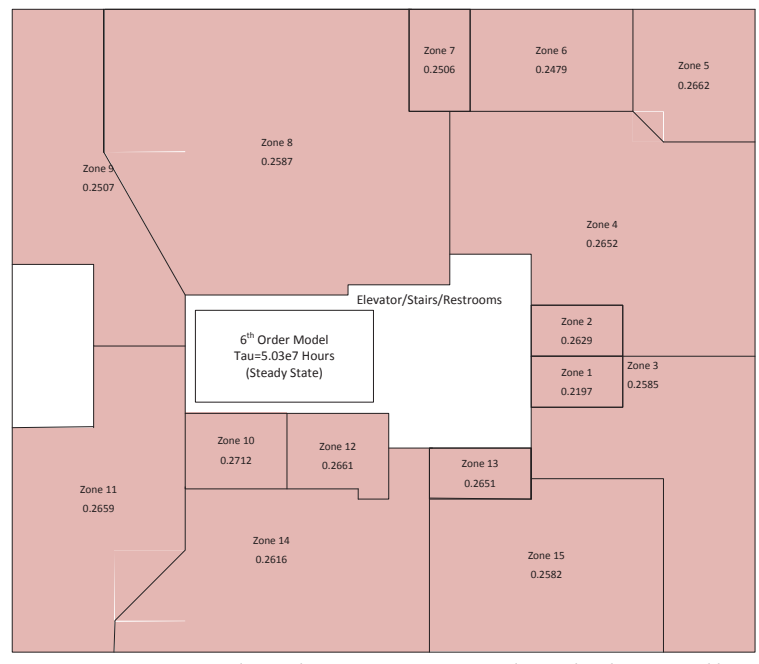

Figure 13: Modes shape associated with the smallest eigenvalue, $7^{\text {th }}$ floor model

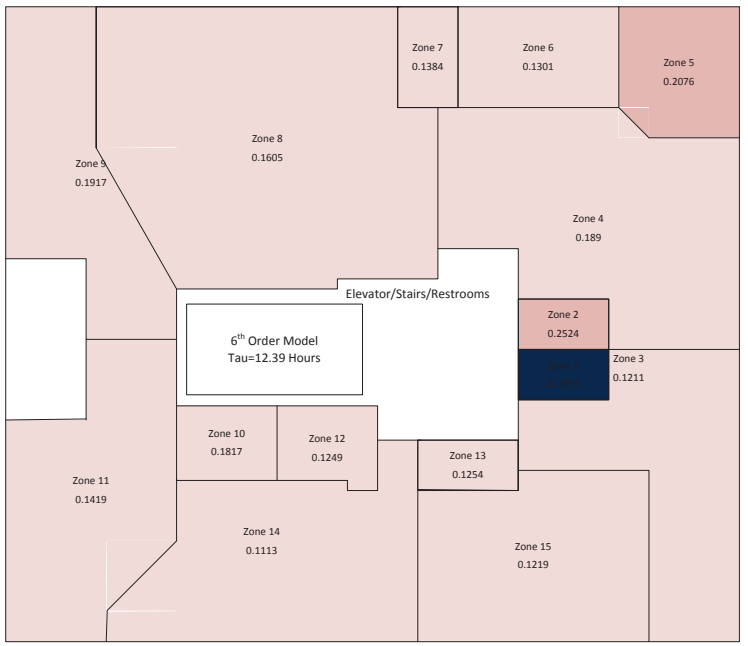

Figure 14: Mode shape associated with a time constant of 12.39 hours, $7^{\text {th }}$ floor model

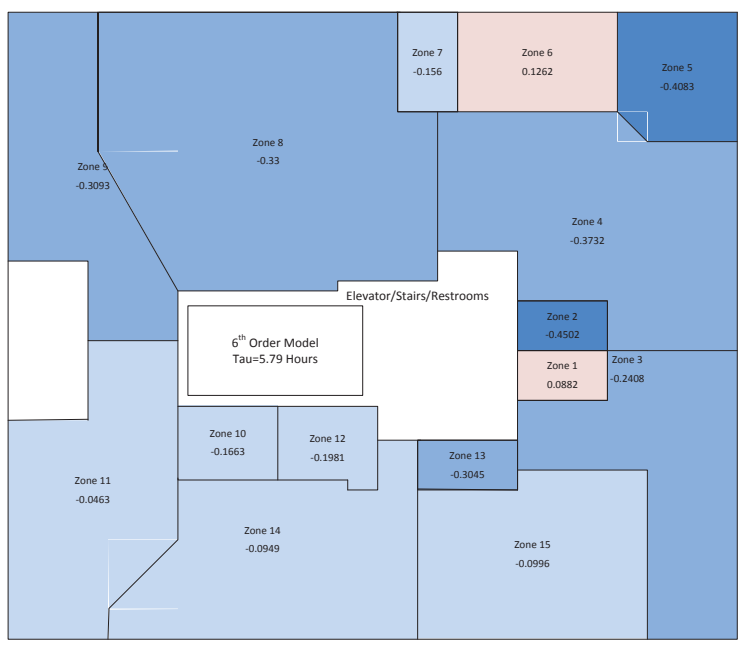

Figure 15: Mode shape associated with a time constant of 5.79 hours, $7^{\text {th }}$ floor model 


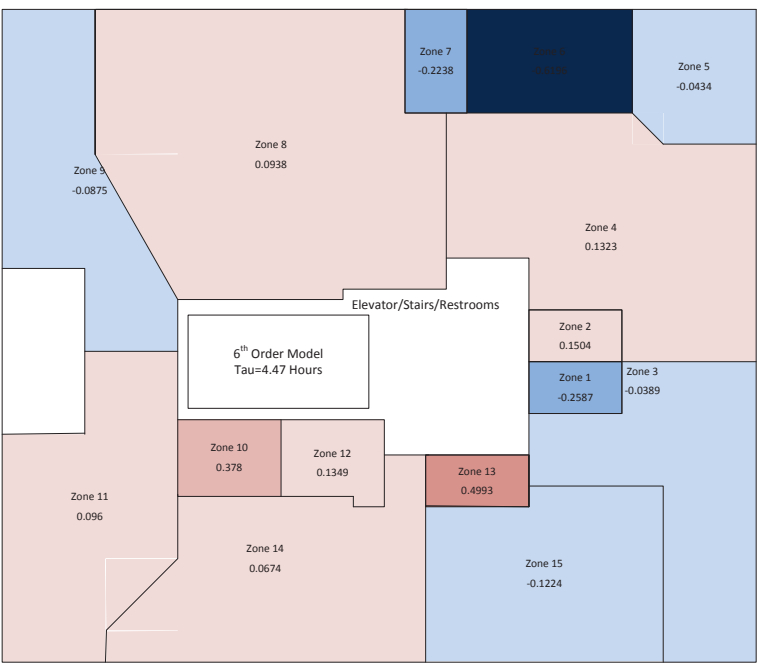

Figure 16: Mode shape with a time constant of 4.47 hours, $7^{\text {th }}$ floor model

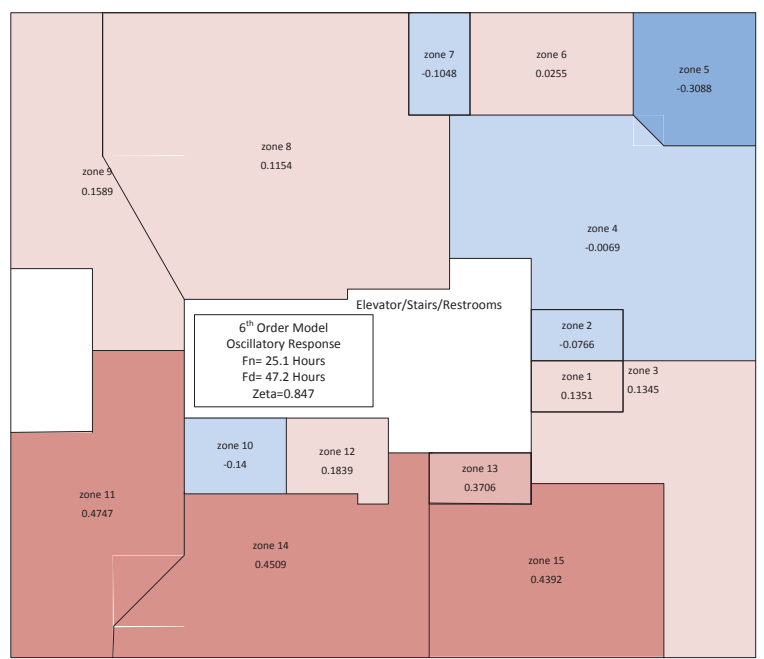

Figure 17: Mode shape of the oscillatory response, period of oscillation, 1.97 days, $7^{\text {th }}$ floor model

\section{Interpretation of Mode Shapes}

Modal analysis is often referred to as a decomposition method. In other words, the actual response of the system is a linear combination of the modal responses. This is the basis for interpreting the mode shapes and time constants. Examining the time constants, we divide the modes into three different domains: steady state, daily and hourly time scales.

The eigenvalues associated with the mode in Figure 13 is essentially zero (4 orders of magnitude smaller than the other eigenvalues). This corresponds to the "rigid body mode" that arises in structural modal analysis. The simplest interpretation is that this is the relative temperature distribution if the system were left undisturbed for a long period of time. We refer to this as the "steady state mode". Note that the mode shape in Figure
13 implies that the temperatures will equilibrate over time, which is an intuitively pleasing result.

Figure 14 shows a mode shape with an associated time constant that is 12.39 hours. This corresponds to a $5 \%$ settling time (3 time constants) of approximately 36 hours. It's not clear where these dynamics are arising, but it's interesting to note that one zone (zone 1) appears with an opposing sign. In other words, as the rest of the system warms up with these dynamics, zone 1 cools down (or vice versa). Also note that color intensity implies the relative participation of that zone in the mode. It appears that this mode almost exclusively involves zone 1 with secondary participation with zones 2 and 5 (acting in opposite signs).

Figures 15 and 16 are intra-day modes with time constants of 5.79 and 4.47 hours, respectively. Clearly these are the main modes that describe the actions of the system throughout the day. Figure 15 shows a response dominated by zones 2,4 and 5 and an out-of phase participation with zones 1 and 6 . By contrast, Figure 16 shows much broader participation by all zones, yet zone 6 is the dominant participant.

Finally Figure 17 shows a mode shape associated with the oscillatory mode. The period of oscillation is approximately 2 days with the corner of zone 5 (South corner of the building) being out of phase with the North corner (zone 11).

\section{Implications for thermal energy storage}

There is an important and critical trade-off in the discussion of thermal energy storage in buildings. On one hand, to make the process as useful as possible to utilities, the storage mechanism should respond quickly to outside signals. In other words, if a wind farm suddenly stops producing because of a lull in the wind, it would be good for buildings to coast their HVAC systems immediately to a lower demand on the grid while the utilities are bringing other generation resources on line. On the other hand, longer time constants imply larger thermal mass which means more energy storage with smaller changes in temperature.

The steady state mode (Figure 13) encompasses the entire thermal mass of the building, but it's long (approaching infinity) time constant implies that this mode does not represent a good mechanism for controllable energy storage. Of the modes discovered in this example, the intra-day modes (figures 15 and 16) seem to offer the most potential for controllable or dispatchable energy storage. Figure 15 in particular, with broad participation, implying that a change in stored thermal energy would affect most zones in a similar manner, and relative short time constant, shows high promise for thermal energy storage on reasonable time scales. 


\section{$\underline{5^{\text {th }} \text { Floor }}$}

In the previous section, we saw how a $6^{\text {th }}$ order model was used to decompose the response that was described by 15 temperature points. The $5^{\text {th }}$ floor of the building is characterized by far more walled offices around the perimeter and far more individual temperature measuring points at 38 .

Using the same process outlined in previous sections, it was found that a $5^{\text {th }}$ order state space model adequately describes the observed behavior of the system and this results in 3 real eigenvalues and one complex conjugate pair. Figures 1821 show the mode shapes of these responses.

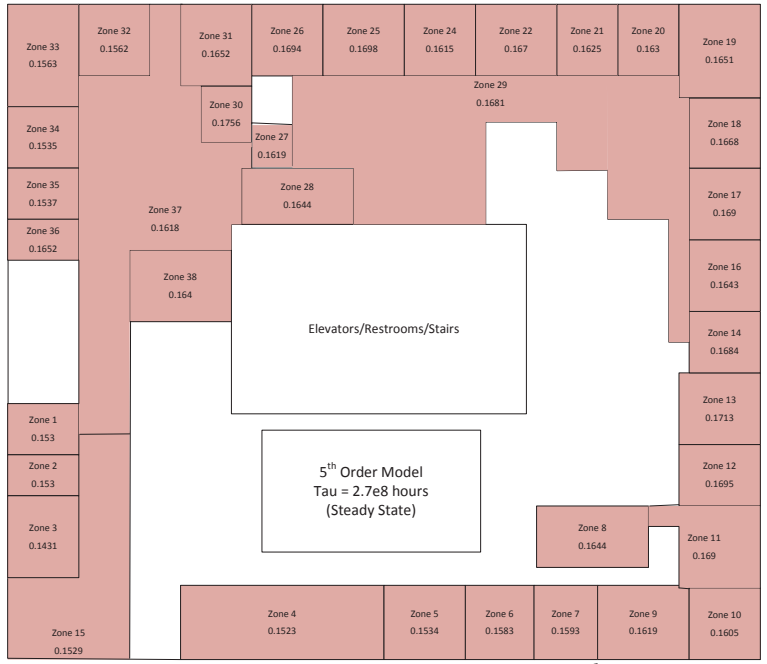

Figure 18: Steady state mode shape for the $5^{\text {th }}$ order model of the $5^{\text {th }}$ floor.

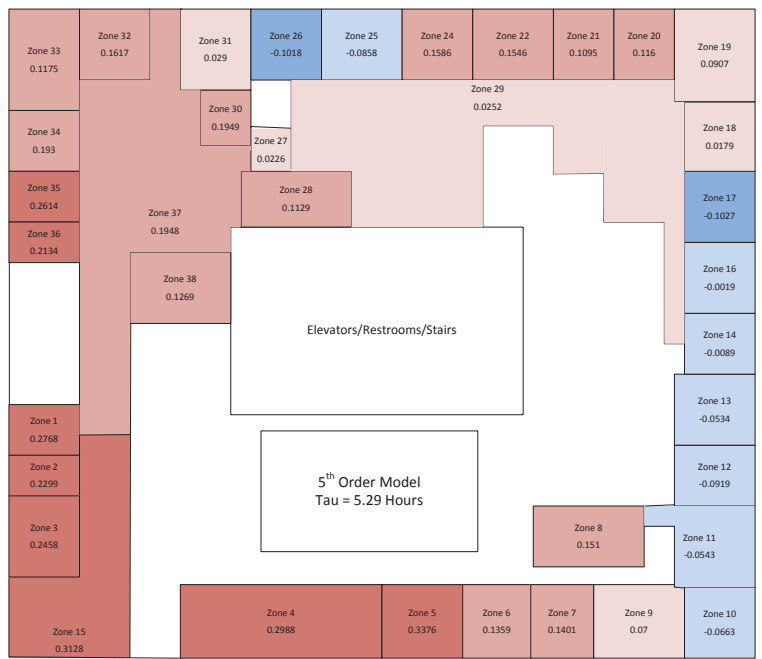

Figure 19: Mode shape associated with a time constant of 5.29 hours $\left(5^{\text {th }}\right.$ order model - Floor 5$)$

Figure 18 shows the steady state, uniform response, as we saw in the previous section. Figure 19 shows an interesting pattern in which most of the measured zones appear to participate and two regions are out of phase with the rest of the building. The time constant (5.29 hours) suggests that it represents an important part of how the HVAC system works throughout the day. Also note that the blue-colored zones on the right part of the figure are facing southwest and receive significantly higher solar loads than the other facades.

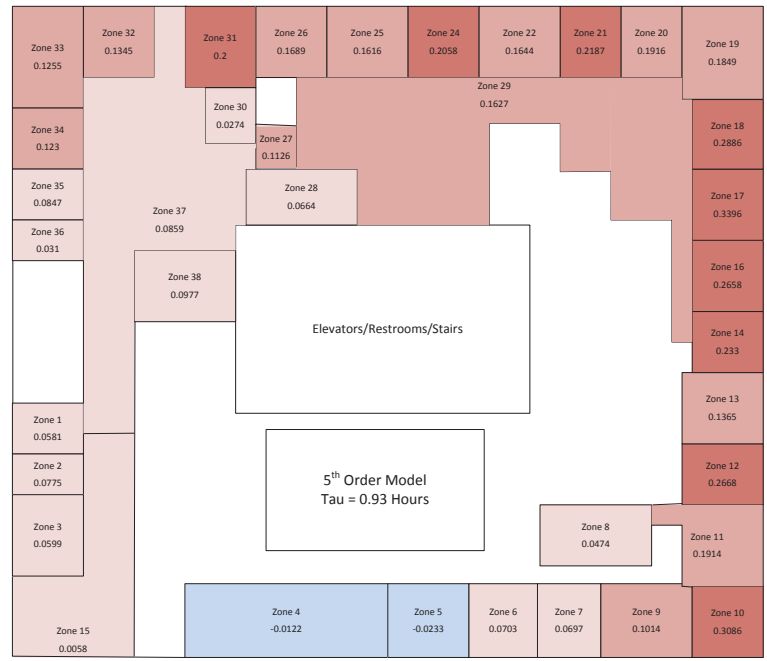

Figure 20: Mode shape associated with a time constant of 0.93 hours $\left(5^{\text {th }}\right.$ order model - Floor 5$)$

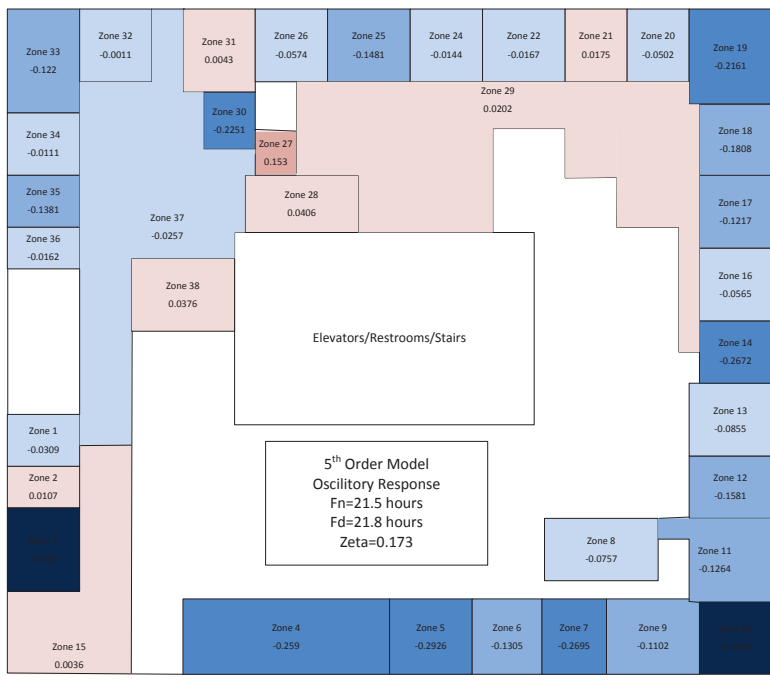

Figure 21: Mode shape of an oscillatory response, 21.8 hour period. ( $5^{\text {th }}$ order model - Floor 5$)$

Figure 20 shows a relatively fast mode, with a time constant less than an hour, involving many of the offices at varying levels of participation. It is unclear how to interpret this particular mode.

Finally, Figure 21 shows an oscillatory mode with a period of response that is very close to the daily cycle. Note that the modeling process used an occupancy signal as one of the inputs, so this response represents a pattern seen in the data that could not be correlated directly to that input pattern. Therefore, we 
consider it likely that this response is related to the variable solar loading experienced throughout the day.

\section{CONCLUSIONS AND FUTURE WORK}

This paper demonstrates an innovative approach to decomposing building temperature responses in an effort to better understand the thermal mass distribution of a structure. The method was demonstrated to be effective and accurate by using simulated data from a system whose structure is known. The method is also demonstrated for actual building temperature data from a complex multi-story, multi-zone commercial building. While the resulting modal decomposition provided some meaningful insight, it is clear that further experience and development is necessary before this can be considered a useful and versatile tool in analyzing system behavior. The areas that are being further considered are:

- Inclusion of HVAC state data into the modeling and system identification processes

- Inclusion of building construction and usage information to help inform the interpretation of the mode shapes

- Apply the methodology on longer data records and investigate the seasonal variations and the impact of cooling vs. heating seasons.

- Active experiments on the building where the system is allowed to coast or the temperature set points are suddenly changed.

Clearly, the modal-analysis of multi-zone building temperature data holds promise in developing low-order linear models that explain significant components of building thermal behavior. The results also have implications and applications to optimal control, fault detection, and demand response.

\section{ACKNOWLEDGMENTS}

This work was supported by the U.S. Department of Energy under DOE Idaho Operations Office Contract DEAC07-05ID14517, and performed as part of the Center for Advanced Energy Studies, and the Instrumentation, Control, and Intelligent Systems (ICIS) Distinctive Signature of Idaho National Laboratory. The United States Government retains, and by accepting the article for publication, the publisher acknowledges that the United States Government retains, a nonexclusive, paid-up, irrevocable, worldwide license to publish or reproduce the published form of this work, or allow others to do so, for United States Government purposes.

\section{REFERENCES}

Ahmad, M. and C. Culp (2006). "Uncalibrated Building Energy Simulation modeling results." HVAC\&R Research 12(4): 1141-1155.

Dincer, I. and M. Rosen (2008). "Exergetically Efficient Thermal Energy Storage Systems for Sustainable Buildings." ASHRAE Transactions 114(1): 98-107.

EERE (2012). Getting Started with Energy Plus: Basic Concepts Manual. L. B. N. Laboratory. http://apps1.eere.energy.gov/buildings/energyplus/pdfs /gettingstarted.pdf, Department of Energy.

Kreider, J. and J. Haberl (1994). "Predicting hourly building energy use: The great energy predictor shootout Overview an ddiscussion of results." $\underline{\text { ASHRAE }}$ Transation: Symposia 100(2): 1104-1118.

Lefebvre, G. (1997). "Modal-based simulaiton of the thermal behavior of a building: the $\mathrm{m} 2 \mathrm{~m}$ sfotware." Energy and Buildings 25: 19-30.

Ljung, L. (1997). System Identification Toolbox: For Use with MaATLAB(r). Cambridge, MA, Mathworks, Inc: 274.

MacCracken, M. (2004). "Thermal energy storage in sustainable buildings." ASHRAE Journal 46(9): S39S41.

MacKay, D. (2009). Sustainable Energy - Without the Hot Air. Cambridge, England, UIT Cambridge Ltd.: 380.

Matiasovsky, P. (2006). Application of modal analysis to identification of building thermal parameters. Thermophysics 2006.

Mejri, O., E. P. D. Barrio, et al. (2011). "Energy performance assessment of occupied buildings using model identifcation techniques." Energy and Buildings 43: 285-299.

Rabl, A. (1988). "Parameter-Estimation in Buildings - Methods for Dynamic Analysis of Measured Energy Use." Journal of Solar Energy Engineering-Transactions of the Asme 110(1): 52-66.

Rao, S. (2010). Mechanical Vibrations, Prentice Hall.

Wijayasekara, O. L. D., M. Manic, et al. (2012). Computational Intelligence based Anomaly Detection for Building ENergy Management Systems. 5th International Symposium on Reslient Control Systems, Salt Lake City, UT, IEEE. 